\title{
Revista Colombiana de

\section{Utilidad del factor de crecimiento fibroblástico 23 en la prevención de enfermedades cardiovasculares en pacientes con enfermedad renal crónica}

\author{
Ibón Santos ${ }^{\mathrm{a}, \mathrm{b}, *}$, María Arango ${ }^{\mathrm{a}, \mathrm{b}}$ y Andrés Pérez ${ }^{\mathrm{a}, \mathrm{b}}$ \\ a Grupo de Investigación Semillero Seres, Facultad de Medicina, Corporación Universitaria Remington - Uniremington, Medellín, \\ Colombia \\ ${ }^{\mathrm{b}}$ Facultad de Medicina, X Semestre. Corporación Universitaria Remington - Uniremington, Medellín, Colombia
}

Recibido el 30 de septiembre de 2015; aceptado el 1 de diciembre de 2015

Disponible en Internet el 16 de enero de 2016

\section{PALABRAS CLAVE \\ Enfermedad \\ cardiovascular; \\ Metabolismo; \\ Calcio; \\ Biomarcadores}

\section{KEYWORDS}

Cardiovascular

disease;

Metabolism;

Calcium;

Biomarkers

\begin{abstract}
Resumen El factor de crecimiento de fibroblastos 23 es una proteína recientemente descubierta, producida por osteocitos y cuya función principal es regular la homeostasis del fosfato y del calcio en el metabolismo mineral óseo. Algunos estudios han encontrado evidencia que sugiere la asociación entre el aumento de las concentraciones del factor de crecimiento fibroblástico 23 y la aparición de diferentes patologías cardiovasculares como: la hipertrofia ventricular izquierda, la falla cardiaca, el choque cardiogénico, la ateroesclerosis, la calcificación vascular, la fibrilación auricular y el accidente cerebrovascular. Sin embargo, son necesarios más estudios para esclarecer el rol de esta hormona y las patologías antes mencionadas.

( $) 2015$ Sociedad Colombiana de Cardiología y Cirugía Cardiovascular. Publicado por Elsevier España, S.L.U. Este es un artículo Open Access bajo la licencia CC BY-NC-ND (http:// creativecommons.org/s/by-nc-nd/4.0/).
\end{abstract}

Usefulness of fibroblast growth factor 23 for the prevention of cardiovascular diseases in patients with chronic kidney disease

Abstract Fibroblast growth factor 23 is a recently discovered protein produced by osteocytes with the primary role of regulating calcium and phosphate homeostasis in the bone and mineral metabolism. Some studies have found evidence suggesting an association between an increase in the concentrations of fibroblast growth factor 23 and an emergence of different cardiovascular pathologies, such as left ventricular hypertrophy, cardiac failure, cardiogenic shock,

\footnotetext{
* Autor para correspondencia. Dirección postal: Carrea 19 \# 35-51 Medellín, Colombia.

Correo electrónico: ibon1985@hotmail.com (I. Santos).
} 
atherosclerosis, vascular calcification, atrial fibrillation and cerebrovascular accidents. However, further research is required to establish the role of this hormone and the aforementioned conditions.

(C) 2015 Sociedad Colombiana de Cardiología y Cirugía Cardiovascular. Published by Elsevier España, S.L.U. This is an open access article under the CC BY-NC-ND license (http:// creativecommons.org/licenses/by-nc-nd/4.0/).

\section{Introducción}

La familia de los factores de crecimiento de los fibroblastos está formado por 23 proteínas, las cuales regulan la proliferación, la migración, la diferenciación y la supervivencia celular ${ }^{1}$. El factor de crecimiento fibroblástico 23 es el integrante más recientemente descubierto de esta estirpe, el cual aumenta su concentración en pacientes con la enfermedad renal crónica, siendo el principal regulador de la homeostasis de fosfato en ellos, al aumentar la fosfaturia en el túbulo contorneado proximal, actuando sobre los transportadores de sodio-fosfato. Además, disminuye las concentraciones circulantes de 1,25-dihidroxivitamina $\mathrm{D}_{3}$ al inhibir la $\alpha$-1-hidroxilasa renal, y reduce la concentración de la hormona paratiroidea, repercutiendo directamente todas estas acciones en el aparato cardiovascular ${ }^{2-6}$.

\section{Relación fisiopatológica del metabolismo mineral ósea, el factor de crecimiento fibroblástico 23 y la enfermedad renal crónica}

El fósforo, el calcio, la vitamina D, la hormona paratiroidea y el factor de crecimiento fibroblástico 23 son los implicados en la homeostasis mineral ósea, las cuales se alteran cuando se instaura la enfermedad renal crónica ${ }^{7}$. Tras el inicio de esta, el factor de crecimiento fibroblástico 23 aumenta su concentración sérica entre 100 y 1.000 veces más ${ }^{8}$, al ser sintetizado por los osteoblastos y en menor medida por osteocitos, debido al incremento de las concentraciones de fósforo que el riñón enfermo no puede eliminar ${ }^{9}$.

A nivel renal, el factor de crecimiento fibroblástico 23 estimula la fosfaturia, al unirse a su receptor $\alpha$-Klotho en el túbulo contorneado proximal, el cual actúa como un correceptor que incrementa la afinidad del complejo ligando/receptor, para producir una disminución de los cotransportadores sodio/fósforo tipo 2, lo que aumenta la excreción renal de fósforo ${ }^{10}$. Por otra parte, actúa sobre la $\alpha$-1-hidroxilasa renal haciendo que disminuya su actividad, y por ende la síntesis de calcitriol (forma biológicamente activa de la vitamina $\mathrm{D}$ ), lo que produce una disminución de la absorción de calcio y de fósforo a nivel entérico, motivo por el que se estimula la liberación de la hormona paratiroidea como mecanismo de contrarregulador de la hipocalcemia, además de la propia hiperfosfatemia ${ }^{11,12}$.

Como es sabido, la hormona paratiroidea produce resorción a nivel óseo, estimulando la liberación de calcio y de fósforo. A nivel renal actúa reabsorbiendo el calcio en el túbulo distal, pero aumenta la excreción de fósforo en el túbulo proximal, además, de estimular la producción de $\alpha-1$ hidroxilasa para aumentar la absorción de calcio entérico ${ }^{13}$.
No obstante, el factor de crecimiento fibroblástico 23 actúa directamente inhibiendo la liberación de la hormona paratiroidea, aunque este efecto no se hace tan evidente en las paratiroides de los pacientes con la enfermedad renal crónica, porque en estos disminuyen la cantidad de receptores del factor de crecimiento fibroblástico 23 en dichas glándulas.

Como es lógico, no solo la hiperfosfatemia inducida por la enfermedad renal crónica es la única variante que interviene en la síntesis del factor de crecimiento fibroblástico 23, ya que altas concentraciones de calcitriol y de la hormona paratiroidea, como puede ser el caso de un hiperparatiroidismo secundario, aumentan los niveles de factor de crecimiento fibroblástico $23^{14}$. Por todo ello es que el factor de crecimiento fibroblástico 23 puede ser un nuevo blanco terapéutico o un predictor de la enfermedad renal crónica y las enfermedades cardiovasculares, el cual debe ser investigado ${ }^{15}$.

\section{Hipertrofia ventricular izquierda, falla cardiaca y choque cardiogénico}

Recientes estudios han determinado que el factor de crecimiento fibroblástico 23 se une a los receptores "FGF-R1" y "FGF-R3" de los miocardiocitos, a pesar de que en estos, el correceptor $\alpha$-Klotho se expresa en minúsculas proporciones. Esta unión produce un aumento del calcio intracelular, que inicialmente aumenta la contractibilidad y la fracción de eyección cardiaca. Si esta situación llega a prolongarse en el tiempo, como consecuencia podría generarse una hipertrofia ventricular, en especial en los pacientes con enfermedad renal crónica ${ }^{16,17}$.

A pesar de lo anterior, artículos más recientes no apoyan esta teoría dado que otros investigadores no han conseguido aislar el correceptor $\alpha$-Klotho en el miocardio, pero si una isoforma de este, llamada $\gamma$-Klotho y pudiera ser posible que haga el mismo efecto en el tejido cardiaco que el correceptor $\alpha$-Klotho, sin embargo, poco se sabe sobre la proteína $\gamma$-Klotho ${ }^{16,18}$.

Se ha podido determinar que los niveles incrementados del fósforo, y por ende del factor de crecimiento fibroblástico 23, están asociados con el incremento de la masa del ventrículo izquierdo, así como de la hipertrofia ventricular izquierda, principalmente en pacientes con enfermedad renal crónica. Esto ha sido explicado por el aumento en la poscarga cardiaca debida a la reducción, la distensibilidad vascular producida por la calcificación arterial ${ }^{19}$. No obstante, esta relación no queda clara, puesto que en varios estudios realizados en pacientes con enfermedad renal crónica en terapia de hemodiálisis, llegaron a la conclusión que 
la relación del factor de crecimiento fibroblástico 23 y la hipertrofia ventricular izquierda no es estadísticamente significativa, ni tampoco su relación con el aumento del índice de masa del ventricular izquierdo ${ }^{20,21}$.

Por otra parte, se ha apreciado un mayor riesgo de desarrollar la falla cardiaca congestiva en los pacientes con enfermedad renal crónica (sobre todo en los que tienen un estadio según la escala NYHA (New York Heart Association), comprendido entre 2 y 4), que además, tenían aumentado las concentraciones del factor de crecimiento fibroblástico $23^{22,23}$. Incluso se ha podido relacionar el incremento de los niveles de factor de crecimiento fibroblástico 23 con el desarrollo del choque cardiogénico, determinando que a mayor concentración, peor es el pronóstico ${ }^{24}$. Estos datos son respaldados por un gran estudio con gran significancia estadística, donde se evaluaron prospectivamente durante 10 años a 3.017 individuos mayores de 65 años de edad, con la enfermedad renal crónica, determinando que altas concentraciones del factor de crecimiento fibroblástico 23 está asociado con el aumento de la incidencia de falla cardiaca ${ }^{25}$.

El estudio IABP-SHOCK " "Intraaortic Balloon Pump in Cardiogenic Shock II' ', evaluó los resultados de 600 pacientes con choque cardiogénico complicado con infarto agudo de miocardio, los cuales fueron aleatorizados a terapia con balón intraaórtico de contrapulsación o no. A este estudio, se le realizó más tarde un subestudio donde se evaluaron los niveles del factor de crecimiento fibroblástico 23 a 182 pacientes, tanto en sujetos que padecían alguna nefropatía como en los que no. Los niveles séricos fueron cuantificados al momento de la admisión, al segundo y al tercer día, todo ello con el objeto de comprender el papel pronóstico del factor de crecimiento fibroblástico 23 en este tipo de pacientes. Se concluyó que el choque cardiogénico y los altos niveles de factor de crecimiento fibroblástico 23 estaban relacionados con mal pronóstico, pero sólo en población con algún daño renal $^{26}$.

\section{Ateroesclerosis}

Varios estudios han intentado usar los niveles del factor de crecimiento fibroblástico 23 como un posible marcador de ateroesclerosis debido a la existencia de cierta homología estructural entre el factor de crecimiento fibroblástico 15, 19 y el 21 con el factor de crecimiento fibroblástico 23. Esto es importante debido a que los dos primeros factores tienen la facultad de mejorar el metabolismo de los lípidos. Ejemplo de ello son el aumento del tejido adiposo marrón o la disminución de la actividad de la acetil coenzima A carboxilasa 2 hepática y por ende la reducción de los niveles de triglicéridos. Las propiedades de estos dos factores es tal, que la administración terapéutica de estos en ratones demostró una disminución de los niveles de glucosa y triglicéridos, lo que haría pensar que el factor de crecimiento fibroblástico 23 pudiera tener un papel protector frente a la ateroesclerosis al disminuir los niveles de colesterol total y $\operatorname{LDL}^{27-29}$.

Esta teoría está respaldada por un estudio donde participaron 196 pacientes con enfermedad renal crónica terminal en diálisis, en los cuales las placas ateromatosas se forman más rápidamente por diversos factores como: las alteraciones en la homeostasis mineral y la metabolización alterada de los lípidos. Estos pacientes fueron evaluados en busca de una asociación entre los niveles del factor de crecimiento fibroblástico 23 y la estenosis de las arterias carótidas mediante la ecografía y los niveles sanguíneos de colesterol total y LDL. Finalmente, el estudio reportó una relación inversamente proporcional estadísticamente significativa entre los niveles del factor de crecimiento fibroblástico 23 y la cuantificación de la estenosis de las arterias carótidas junto con las concentraciones del colesterol total y del LDL, apoyando la hipótesis anterior de que el factor de crecimiento fibroblástico 23 podría tener un rol en la reducción del colesterol total y del $\mathrm{LDL}^{30}$.

Sin embargo, otros estudios con un número mayor de población estudiada, difiere de los resultados antes mencionados. En un subgrupo de 306 pacientes del estudio PIVUS "Prospective Investigation of the Vasculature in Uppsala Seniors", donde evaluaron la extensión y severidad de las placas ateromatosas formadas en todo el árbol arterial corporal mediante angiografía por resonancia magnética con contraste de gadolinio y la relación con los niveles del factor de crecimiento fibroblástico 23 cuantificados mediante ELISA (Enzyme-Linked ImmunoSorbent Assay), se llegó a la conclusión de que existe una relación directamente proporcional entre los niveles del factor de crecimiento fibroblástico 23 y las placas ateromatosas sobre todo en los pacientes con una tasa de filtración glomerular $<60 \mathrm{ml} / \mathrm{min} / 1,73 \mathrm{~m}^{2}{ }^{31}$.

Así mismo, en un estudio que albergó 2.076 pacientes que fueron sometidos a angiografía coronaria, se pretendía determinar la relación entre los niveles del factor de crecimiento fibroblástico 23 y la severidad y la extensión de la enfermedad arterial coronaria. Finalmente, llegaron a la misma conclusión que el anterior estudio, la existencia de una relación entre los niveles del factor de crecimiento fibroblástico 23 y la severidad y la extensión de la placa ateromatosa, en este caso en los vasos coronarios, particularmente, en pacientes con una tasa de filtración glomerular $<60 \mathrm{ml} / \mathrm{min} / 1,73 \mathrm{~m}^{2} 32$.

Al observar la existencia de la relación entre los niveles del factor de crecimiento fibroblástico 23 y el desarrollo de las placas ateromatosas, un estudio evaluó el uso de niacina en los pacientes con enfermedad renal crónica como medida de la reducción de las concentraciones del factor de crecimiento. Este estudio determinó una reducción de una media de un $11 \%$ de la concentración basal del factor de crecimiento fibroblástico 23 en esta población estudiada, alentando, como este medicamento podría ser una estrategia para disminuir los niveles del factor de crecimiento fibroblástico 23 en pacientes con enfermedad renal crónica, y por ende la disminución de la ateroesclerosis ${ }^{33}$.

Algunas investigaciones consideran que el factor de crecimiento fibroblástico 23 puede estar relacionado, en parte, con la ateroesclerosis subclínica temprana y la injuria cardiaca que pueden llegar a presentar los pacientes que sufren la enfermedad de Kawasaki, no obstante, son necesarias más investigaciones que aclaren la relación con la hormona y el daño endotelial de estos pacientes, siendo un posible marcador precoz de la patología cardiovascular ${ }^{34,35}$.

\section{Calcificación vascular}

Hasta la fecha, no se ha podido determinar el mecanismo molecular fisiopatológico exacto de la calcificación vascular, 
aunque se sugiere que las altas concentraciones de fósforo en los pacientes con la enfermedad renal crónica juega un papel vital en esta patología, así como se asume que el factor de crecimiento fibroblástico 23 es el factor protector al promover la fosfaturia ${ }^{36}$.

En el estudio CRIC "Chronic Renal Insufficiency Cohort", donde participaron 1.501 pacientes con la enfermedad renal crónica con la tasa de filtración glomerular $<60 \mathrm{ml} / \mathrm{min} / 1,73 \mathrm{~m}^{2}$, se evaluó la calcificación de las coronarias y la aorta torácica mediante la tomografía computarizada, encontrándose que los altos niveles del factor de crecimiento fibroblástico 23 no aumentaban la prevalencia ni la severidad de la calcificación vascular, como era de esperar. Además, en este estudio no pudieron encontrar la expresión del correceptor Klotho en el tejido vascular liso, algo que ha sido logrado por otros grupos de investigación, los cuales afirman que hallaron presente el complejo Klothofactor de crecimiento fibroblástico 23 en las muestras de la aorta torácica en 44 pacientes llevados a cirugía cardiaca y en dos trombos de dos pacientes que sufrieron un síndrome coronario agudo ${ }^{37,38}$. Tras los hallazgos obtenidos, los autores del estudio CRIC sugieren que el factor de crecimiento fibroblástico 23 puede intervenir en la prevención de la calcificación vascular, en donde el fósforo debe ser el principal factor en la calcificación de los vasos ${ }^{39}$. Otros estudios apoyan estos resultados, como en un estudio reciente en 434 pacientes coreanos donde se estudiaron variantes polimórficas del gen Klotho y determinaron que dichos polimorfismos están asociados con la estenosis arterial coronaria pero no con la calcificación ${ }^{40}$.

Además, en los pacientes que acuden a hemodiálisis, los ensayos realizados muestran similares resultados. De esta forma, las investigaciones afirman que existe una asociación directa entre los niveles del factor de crecimiento fibroblástico 23 y la calcificación vascular, afirmando incluso que este factor de crecimiento podría jugar un papel protector cardiovascular, por lo menos en esta población, sugiriendo que hacen falta más estudios con cohortes más grandes para comprobar esta hipótesis ${ }^{41,42}$.

\section{Fibrilación auricular}

Se ha podido observar como en ensayos in vitro de cardiomiocitos atriales extraídos del ratón, el factor de crecimiento fibroblástico 23 produce un aumento del tránsito de calcio intracelular a través de los canales de calcio tipo $L$, el cual es almacenado en el retículo endoplasmático, a través de la bomba SERCA $2 \mathrm{a}$, produciendo finalmente una prolongación del potencial de acción y por ende un retraso en la post-despolarización cardiaca. El fosfolamban, una proteína que en su estado natural inhibe la bomba SERCA 2 a, tiende a fosoforilarse en presencia del factor de crecimiento fibroblástico 23, incrementando la actividad de esta bomba y a su vez produciendo un aumento exagerado del almacenamiento de calcio en el retículo endoplasmático, con el riesgo de producirse una arritmia cardiaca. Este tránsito se ha podido bloquear en laboratorio mediante bloqueadores de canales de calcio ${ }^{43,44}$.

Varios estudios como el MESA "The Multi-Ethnic Study of Atherosclerosis" y el CHS "Cardiovascular Health Study" han demostrado la asociación entre el aumento del riesgo de fibrilación auricular y la presencia de altos niveles sanguíneos del factor de crecimiento fibroblástico 23. Ambos estudios suman un total de 7.748 pacientes evaluados $(6.398$ y 1.350, respectivamente), los cuales no sufrían ninguna enfermedad cardiovascular de base y con una media de edad de 64 y 77 años, respectivamente ${ }^{45}$.

Por el contrario, el estudio ARIC "Atherosclerosis Risk in Communities Study" que contó con la participación de 12.349 pacientes, el cual no excluyó a pacientes con enfermedad cardiovascular previa y la media de edad fue de 57 años, no apoyó los datos de los 2 estudios previamente mencionados. Esto puede ser debido a varios factores, como los métodos de cuantificación del factor de crecimiento fibroblástico 23, debido a que fueron diferentes en los estudios, los criterios de exclusión e inclusión, así como del seguimiento, pues en el estudio ARIC fue de 17 años mientras que en el MESA y el CHS fue de 8 años.

Finalmente, se realizó un metaanálisis con los resultados de los 3 estudios concluyendo una asociación estadísticamente significativa entre los niveles elevados del factor de crecimiento fibroblástico 23 y el aumento del riesgo de fibrilación auricular ${ }^{46}$.

\section{Accidente cerebrovascular}

Pocos estudios han evaluado la relación entre los niveles circulantes del factor de crecimiento fibroblástico 23 y la asociación del accidente cerebrovascular, a pesar que hay una fuerte correlación entre los niveles elevados de la hormona y la enfermedades cardiovasculares como: la falla cardiaca, la ateroesclerosis y la fibrilación auricular. Debido en gran parte a esta última y su gran relación con el accidente cerebrovascular, un ensayo reciente diseñado como casos y controles, evaluó 1.551 pacientes, 615 clasificados como casos que desarrollaron un accidente cerebrovascular y 936 controles que fueron seleccionados de un estudio previo llamado REGARDS "Reasons for Geographic and Racial Differences in Stroke" que estudió aproximadamente a 30.000 pacientes y sus características con el accidente cerebrovascular, para evidenciar el papel del factor de crecimiento fibroblástico 23 en la génesis del accidente cerebrovascular. Estos hallaron que las concentraciones elevadas del factor estaban asociadas a un mayor riesgo cardioembólico, pero no al accidente cerebrovascular de origen ateroesclerótico ni hemorrágico ${ }^{47}$.

No obstante, del estudio NOMAS "The Northern Manhattan Study", donde se determinó que los hispanos-caribeños residentes en la comunidad del alto Manhattan (Estados Unidos), sufren accidentes cerebrovasculares a edad más temprana que los negros y los blancos residentes en la misma comunidad, se sacó un subgrupo de estudio de 2.525 pacientes que fueron seguidos una media de 12 años para detectar en ellos la relación del accidente cerebrovascular con los niveles del factor de crecimiento fibroblástico 23, determinándose que los niveles elevados de este último confiere el doble de riesgo de accidente cerebrovascular, en especial sobre el hemorrágico, independientemente, de la enfermedad renal crónica ${ }^{48}$. Otros estudios han mostrado evidencia similar en referencia al accidente cerebrovascular, en particular al hemorrágico ${ }^{49}$. No obstante, pocos estudios se han realizado en esta área, por lo que se necesitan más ensayos 
para comprender la prevalencia de los diferentes accidentes cerebrovasculares y su relación con el factor de crecimiento fibroblástico $23^{50}$.

\section{Conclusiones}

En vista de la discrepancia entre las recientes investigaciones, se puede concluir que hacen falta más ensayos para esclarecer si el factor de crecimiento fibroblástico 23 tiene implicación directa sobre la hipertrofia ventricular izquierda, o si esta es debida a las altas concentraciones del fósforo y la calcificación vascular que produce, aumentando por ende la poscarga, sin embargo, las investigaciones son claras al evidenciar la falla cardiaca y el choque cardiogénico y las altas concentraciones circulantes del factor de crecimiento fibroblástico 23. Por otra parte, la evidencia científica tiende a relacionar los niveles del factor de crecimiento fibroblástico 23 en los pacientes con la enfermedad renal crónica y la extensión y la gravedad de la placa ateromatosa. Teniendo esto en cuenta, se deben direccionar más estudios para evaluar las posibles intervenciones sobre el factor, puesto que pudiera ser un objetivo terapéutico viable para disminuir el riesgo cardiovascular, sobre todo en la población con la enfermedad renal crónica. Igualmente se puede observar, que hay clara relación entre los niveles del factor de crecimiento fibroblástico 23 y de la calcificación vascular, lo cual puede ser explicado por un feed-back positivo, es decir, a mayores niveles de fósforo, mayores niveles del factor de crecimiento fibroblástico 23, con el objetivo de eliminar este electrolito del organismo, previniendo así su efecto deletéreo sobre el sistema vascular. Referente a la fibrilación auricular, parece no haber discusión, ya que el metaanálisis antes mencionado concluye que hay una asociación directa entre los niveles del factor de crecimiento fibroblástico 23 y de la fibrilación auricular, pero queda por aclarar si el control del factor de crecimiento fibroblástico 23 puede ser blanco terapéutico para poder, al menos en parte, disminuir el riesgo de la fibrilación auricular. Por último, y aunque hay pocos estudios que evalúan la asociación entre el factor de crecimiento fibroblástico 23 y el accidente cerebrovascular, estos parecen indicar que puede haber una relación causal entre la aparición de enfermedades de origen cardioembólico y el aumento de las concentraciones del factor de crecimiento fibroblástico 23, no obstante, otro estudio en la población hispano-caribeña ha evidenciado que el aumento de las concentraciones del factor de crecimiento fibroblástico 23 confiere el doble de riesgo de accidente cerebrovascular principalmente hemorrágico.

Es claro, que hacen falta más estudios para obtener la evidencia concluyente sobre un posible y novedoso marcador de la enfermedad cardiovascular, en especial, en pacientes que padecen de enfermedad renal crónica.

\section{Responsabilidades éticas}

Protección de personas y animales. Los autores declaran que para esta investigación no se han realizado experimentos en seres humanos ni en animales.
Confidencialidad de los datos. Los autores declaran que en este artículo no aparecen datos de pacientes.

Derecho a la privacidad y consentimiento informado. Los autores declaran que en este artículo no aparecen datos de pacientes.

\section{Conflicto de intereses}

Los autores declaran no tener ningún conflicto de intereses.

\section{Bibliografía}

1. Eswarakumar VP, Lax I, Schlessinger J. Cellular signaling by fibroblast growth factor receptors. Cytokine Growth Factor Rev. 2005; 16:139-49.

2. Ben-Dov IZ, Galitzer H, Lavi-Moshayoff V, Goetz R, Kuro-o M, Mohammadi $\mathrm{M}$, et al. The parathyroid is a target organ for FGF23 in rats. J Clin Invest. 2007;117:4003-8.

3. Kurosu H, Ogawa Y, Miyoshi M, Yamamoto M, Nandi A, Rosenblatt $\mathrm{KP}$, et al. Regulation of fibroblast growth factor-23 signaling by Klotho. J Biol Chem. 2006;281:6120-3.

4. Shimada T, Mizutani S, Muto T, Yoneya T, Hino R, Takeda S, et al. Cloning and characterization of FGF23 as a causative factor of tumor-induced osteomalacia. Proc Natl Acad Sci USA. 2001;98:6500-5.

5. Urakawa I, Yamazaki Y, Shimada T, Iijima K, Hasegawa H, Okawa $\mathrm{K}$, et al. Klotho converts canonical FGF receptor into a specific receptor for FGF23. Nature. 2006;444:770-4.

6. Wolf M. Forging forward with 10 burning questions on FGF23 in kidney disease. J Am Soc Nephrol. 2010;21:1427-35.

7. Mejía N, Roman-García P, Miar AB, Tavira B, Cannata-Andía JB. El complejo escenario de las alteraciones de metabolismo óseo y mineral en la enfermedad renal crónica. Nefrología. 2011;31:514-9.

8. Agarwal I, Ide N, Ix JH, Kestenbaum B, Lanske B, Schiller NB, et al. Fibroblast growth factor-23 and cardiac structure and function. J Am Heart Assoc. Blackwell Publishing Inc. 2014;3.

9. Jüppner $H$, Wolf $M$, Salusky IB. FGF-23: More than a regulator of renal phosphate handling? Journal of Bone and Mineral Research. 2010:2091-7.

10. Jüppner H, Wolf M. aKlotho: FGF23 coreceptor and FGF23regulating hormone. Journal of Clinical Investigation. 2012: 4336-9.

11. Jüppner H. Phosphate and FGF-23. Kidney Int Suppl. 2011: S24-7.

12. Silver J, Naveh-Many T. FGF-23 and secondary hyperparathyroidism in chronic kidney disease. Nat Rev Nephrol. Nature Publishing Group. 2013;9:641-9.

13. Parathyroid N, Parathyroid N, Growth C, Growth C. Cell Biology of Parathyroid Gland Hyperplasia in Chronic Renal Failure. Differences. 2000:1141-52.

14. Sánchez-González MC, Salanova L, Ruano P. FGF-23: ¿solo regulador del metabolismo del fósforo o algo más? Reumatol Clin. 2011;7 Suppl. 2:5-7.

15. Russo D, Battaglia Y. Clinical Significance of FGF-23 in Patients with CKD. Int J Nephrol. 2011;2011:364-890.

16. Touchberry CD, Green TM, Tchikrizov V, Mannix JE, Mao TF, Carney BW, et al. FGF23 is a novel regulator of intracellular calcium and cardiac contractility in addition to cardiac hypertrophy. Am J Physiol Endocrinol Metab. 2013;304:E863-73.

17. Faul C, Amaral AP, Oskouei B, Hu MC, Sloan A, Isakova T, et al. FGF23 induces left ventricular hypertrophy. J Clin Invest. 2011;121:4393-408. 
18. Kovesdy CP, Quarles LD. Fibroblast growth factor-23: what we know, what we don't know, and what we need to know. Nephrol Dial Transplant. 2013;28:2228-36.

19. Scialla JJ, Wolf M. Roles of phosphate and fibroblast growth factor 23 in cardiovascular disease. Nat Rev Nephrol. Nature Publishing Group. 2014;10:268-78.

20. Unsal A, Budak SK, Koc Y, Basturk T, Sakaci T, Ahbap E, et al. Relationship of fibroblast growth factor 23 with left ventricle mass index and coronary calcificaton in chronic renal disease. Kidney Blood Press Res. 2012;36:55-64.

21. Sany D, Elsawy AE, Aziz A, Elshahawy Y, Ahmed H, Aref H, et al. The value of serum FGF-23 as a cardiovascular marker in HD patients. Saudi J Kidney Dis Transpl. 2014;25:44-52.

22. Scialla JJ, Xie H, Rahman M, Anderson AH, Isakova T, Ojo A, et al. Fibroblast growth factor-23 and cardiovascular events in CKD. J Am Soc Nephrol. 2014;25:349-60.

23. Imazu M, Takahama $H$, Asanuma $H$, Funada A, Sugano $Y$, Ohara T, et al. Pathophysiological impact of serum fibroblast growth factor 23 in patients with nonischemic cardiac disease and early chronic kidney disease. AJP Hear Circ Physiol. 2014;307:H1504-11.

24. Pöss J, Mahfoud F, Seiler S, Heine GH, Fliser D, Böhm M, et al. FGF-23 is associated with increased disease severity and early mortality in cardiogenic shock. Eur Hear journal Acute Cardiovasc Care. 2013;2:211-8.

25. Ix JH, Katz R, Kestenbaum BR, De Boer IH, Chonchol M, Mukamal KJ, et al. Fibroblast growth factor-23 and death, heart failure, and cardiovascular events in community-living individuals: CHS (Cardiovascular Health Study). J Am Coll Cardiol. 2012;60:200-7.

26. Fuernau G, Pöss J, Denks D, Desch S, Heine GH, Eitel I, et al. Fibroblast growth factor 23 in acute myocardial infarction complicated by cardiogenic shock: a biomarker substudy of the Intraaortic Balloon Pump in Cardiogenic Shock II (IABP-SHOCK II) trial. Crit Care. 2014;18:1-9.

27. Huang X, Yang C, Luo Y, Jin C, Wang F, McKeehan WL. FGFR4 prevents hyperlipidemia and insulin resistance but underlies high-fat diet-induced fatty liver. Diabetes. 2007;56:2501-10.

28. Kharitonenkov A, Shiyanova TL, Koester A, Ford AM, Micanovic R, Galbreath EJ, et al. FGF-21 as a novel metabolic regulator. J Clin Invest. 2005;115:1627-35.

29. Tomlinson E, Fu L, John L, Hultgren B, Huang X, Renz M, et al. Transgenic mice expressing human fibroblast growth factor-19 display increased metabolic rate and decreased adiposity. Endocrinology. 2002;143:1741-7.

30. Ashikaga E, Honda H, Suzuki H, Hosaka N, Hirai Y, Sanada $D$, et al. Impact of fibroblast growth factor 23 on lipids and atherosclerosis in hemodialysis patients. Ther Apher Dial. 2010;14:315-22.

31. Mirza MAI, Hansen T, Johansson L, Ahlström H, Larsson A, Lind L, et al. Relationship between circulating FGF23 and total body atherosclerosis in the community. Nephrol Dial Transplant. 2009;24:3125-31.

32. Xiao Y, Peng C, Huang W, Zhang J, Xia M, Zhang Y, et al. Circulating Fibroblast Growth Factor 23 Is Associated with Angiographic Severity and Extent of Coronary Artery Disease. PLoS One. 2013;8.

33. Rao M, Steffes M, Bostom A, Ix JH. Effect of niacin on FGF23 concentration in chronic kidney disease. Am J Nephrol. S. Karger AG. 2014;39:484-90.

34. Falcini F, Masi L, Leoncini G, Franceschelli F, Vitale A, Capannini $S$, et al. ROLE of FGF23 IN kawasaki disease (KD): A possible predictor of subclinical atherosclerosis. Arthritis Rheum. 2009;60:1538.
35. Falcini F, Masi L, Leoncini G, Franceschelli F, Capannini S, La Torre F, et al. Identification of a new polymorphism of the FGF23 GENE: A possible predictor of coronary damage in Kawasaki Disease (KD). Clin Exp Rheumatol. 2011;29:398.

36. Zhu D, Mackenzie NCW, Millan JL, Farquharson C, MacRae VE. A protective role for FGF-23 in local defence against disrupted arterial wall integrity? Mol Cell Endocrinol. Elsevier Ireland Ltd. 2013;372:1-11.

37. Van Venrooij NA, Pereira RC, Tintut Y, Fishbein MC, Tumber N, Demer LL, et al. FGF23 protein expression in coronary arteries is associated with impaired kidney function. Nephrol Dial Transplant. Oxford University Press. 2014;29:1525-32.

38. Donate-Correa J, Mora-Fernández C, Martínez-Sanz R, MurosDe-Fuentes M, Pérez H, Meneses-Pérez B, et al. Expression of FGF23/KLOTHO system in human vascular tissue. Int J Cardiol. Elsevier Ireland Ltd. 2013;165:179-83.

39. Scialla JJ, Lau WL, Reilly MP, Isakova T, Yang H-Y, Crouthamel $\mathrm{MH}$, et al. Fibroblast growth factor 23 is not associated with and does not induce arterial calcification. Kidney Int. Nature Publishing Group. 2013;83:1159-68.

40. Jo S-H, Kim S-G, Choi YJ, Joo N-R, Cho G-Y, Choi S-R, et al. KLOTHO gene polymorphism is associated with coronary artery stenosis but not with coronary calcification in a Korean population. Int Heart J. 2009;50:23-32.

41. Moldovan D, Moldovan I, Rusu C, Kacso I, Patiu IM, GhermanCaprioara M. FGF-23, vascular calcification, and cardiovascular diseases in chronic hemodialysis patients. Int Urol Nephrol. 2013.

42. Srivaths PR, Goldstein SL, Silverstein DM, Krishnamurthy R, Brewer ED. Elevated FGF 23 and phosphorus are associated with coronary calcification in hemodialysis patients. Pediatr Nephrol. 2011;26:945-51.

43. Kao YH, Chen YC, Lin YK, Shiu RJ, Chao TF, Chen SA, et al. FGF-23 dysregulates calcium homeostasis and electrophysiological properties in HL-1 atrial cells. Eur J Clin Invest. Blackwell Publishing Ltd. 2014;44:795-801.

44. Schwinger RH, Münch G, Bölck B, Karczewski P, Krause EG, Erdmann E. Reduced $\mathrm{Ca}(2+)$-sensitivity of SERCA $2 \mathrm{a}$ in failing human myocardium due to reduced serin-16 phospholamban phosphorylation. J Mol Cell Cardiol. 1999;31:479-91.

45. Mathew JS, Sachs MC, Katz R, Patton KK, Heckbert SR, Hoofnagle $\mathrm{AN}$, et al. Fibroblast growth factor-23 and incident atrial fibrillation: The multi-ethnic study of atherosclerosis (MESA) and the cardiovascular health study (CHS). Circulation. Lippincott Williams and Wilkins. 2014;130:298-307.

46. Alonso A, Lopez FL, Matsushita K, Loehr LR, Agarwal SK, Chen LY, et al. Chronic kidney disease is associated with the incidence of atrial fibrillation: The atherosclerosis risk in communities (ARIC) Study. Circulation. 2011;123:2946-53.

47. Panwar B, Jenny NS, Howard VJ, Wadley VG, Muntner P, Kissela BM, et al. Fibroblast Growth Factor 23 and Risk of Incident Stroke in Community-Living Adults. Stroke. 2015;46: 322-8.

48. Wright CB, Dong C, Stark M, Silverberg S, Rundek T, Elkind MSV, et al. Plasma FGF23 and the risk of stroke: The Northern Manhattan Study (NOMAS). Neurology. 2014;82:1700-6.

49. Di Giuseppe R, Kühn T, Hirche F, Buijsse B, Dierkes J, Fritsche A, et al. Plasma fibroblast growth factor 23 and risk of cardiovascular disease: results from the EPIC-Germany case-cohort study. Eur J Epidemiol. 2015;30:131-41.

50. Zou L, Zheng S, Letter by Zou and Zheng Regarding Article. Fibroblast Growth Factor 23 and Risk of Incident Stroke in Community-Living Adults. Stroke. 2015;46, e123-e123. 